\title{
Estructura factorial de la escala "Falta de personal" en trabajadores de una empresa retail peruana
}

\author{
Factor structure of the "Understaffing" scale in workers of a peruvian \\ retail company
}

\author{
Mijail Epifanio Andia Ventura ${ }^{1}$ \\ Universidad Nacional Federico Villarreal \\ Ronald Wilfredo Castillo Blanco ${ }^{2}$ \\ Universidad del Pacífico
}

Recibido: $27-04-21$

Aceptado: $09-05-21$

Publicado: $18-06-21$

\section{Resumen}

La presente investigación tiene como objetivo analizar la estructura interna de la escala "Falta de Personal". Los participantes fueron 201 trabajadores de ambos géneros del sector retail de una empresa peruana. El análisis de estructura interna evidenció un mejor ajuste para el modelo de dos factores correlacionados de la escala, "Falta de mano de obra" y "Falta de experticia", coincidiendo con la definición del constructo realizada por sus creadoras, Hudson y Shen (2015). El análisis de la confiabilidad se realizó a través del método de consistencia interna, obteniéndose valores de $\omega=.77$ y $\omega=.78$, respectivamente. En conclusión, los resultados evidencian soporte a la estructura de dos factores correlacionados.

Palabras clave: falta de personal; falta de mano de obra; falta de experticia; análisis factorial confirmatorio.

\begin{abstract}
The present study aims to analyze the internal structure of the "Lack of Personnel" scale. Participants were 201 workers of both genders from the retail sector of a Peruvian company. The evaluation of the internal structure shows a better fit for the model of two correlated factors of the scale, "Lack of manpower" and "Lack of expertise", coinciding with the definition of the construct made by its creators, Hudson and Shen (2015). The reliability analysis was carried out through the internal consistency method, obtaining values of $\omega=.77$ and $\omega=.78$, respectively. In conclusion, the results show support for the theoretical structure of two correlated factors.
\end{abstract}

Keywords: understaffing; manpower understaffing; expertise understaffing; confirmatory factor analysis.

1 Universidad Nacional Federico Villarreal. Lima, Perú.

Autor para correspondencia: mijail.andia.ventura@gmail.com ORCID: https://orcid.org/0000-0002-0663-5707

2 Universidad del Pacifico. Lima, Perú.

E-mail: rw.castillob@up.edu.pe ORCID: https://orcid.org/0000-0003-2945-3583

(C) Los autores. Este artículo es publicado por la Revista de Investigación en Psicología de la Facultad de Psicología, Universidad Nacional Mayor de San Marcos. Este es un artículo de acceso abierto, distribuido bajo los términos de la licencia Creative Commons Atribución 4.0 Internacional (CC BY 4.0) [https://creativecommons.org/licenses/by/4.0/deed.es] que permite el uso, distribución y reproducción en cualquier medio, siempre que la obra original sea debidamente citada de su fuente original. 
Actualmente, las organizaciones buscan ser más competitivas y como parte de esa orientación hacia la competitividad, constantemente buscan maximizar sus ingresos y minimizar sus costos (Varian, 1998). Dentro de la estructura de costos de una organización, una sección importante viene a estar dada por los costos de personal. Cuando una organización enfrenta un problema económico, ya sea producido por una ineficiente gestión interna o una coyuntura externa, una de las primeras decisiones es la reducción de costos, siendo la reducción de personal una opción (Rodríguez, 2010).

Asimismo, la reducción de personal no implica necesariamente que los objetivos de la organización, en términos de funciones y tareas a realizar, disminuyan, sino que, por el contrario, se mantienen y en algunas oportunidades tienden a aumentar (Rivera, 2017). Es en ese contexto que los trabajadores de la organización que aún se mantienen dentro de la misma, asumen las funciones de quienes fueron retirados de la organización, de tal manera, que, si esta situación se mantiene en el largo plazo, puede generar consecuencias en el bienestar en los trabajadores (Herrera et al., 2017).

Por otro lado, desde la perspectiva de reducción de costos de personal, existen organizaciones que toman la decisión de cambiar la estructura de sus puestos de tal manera que, en un área en particular, si antes se contaba con un "Analista" ahora la nueva estructura del área contará con una posición de jerarquía inferior como un "Asistente" o incluso "Practicantes". Claro está, que el logro de los objetivos del área de trabajo se mantiene, independientemente de la nueva estructura de puestos que posea. Es ahí, que mientras una posición sea más “junior" su nivel de experticia será menor pudiendo este ser un factor que podría comprometer el cumplimiento de los objetivos trazados.

Hudson y Shen (2015) engloban estas dos situaciones bajo el constructo "understaffing", o "falta de personal" traducido al español, el cual debe ser entendido bajo un enfoque multidimensional. La primera dimensión de la "falta de personal" es concebida como el déficit de personas que son necesarias para que un área de trabajo logre sus objetivos estratégicos, a la cual denominan "Manpower understaffing" (Falta de mano de obra). La segunda dimensión de la "falta de personal" es concebida como el déficit de habilidades, conocimientos, experticia en los integrantes de un área de trabajo que son necesarias para lograr los objetivos estratégicos. Esta segunda dimensión recibe el nombre de "Expertise understaffing" (Falta de experticia).

En el año 2015, Hudson y Shen hacen una revisión de la literatura de cómo este constructo ha sido abordado a lo largo de los años, en los que, como veremos, el enfoque de la "falta de personal" ha ido evolucionando. Barker (1968), fue el pionero en investigar cuáles son las consecuencias de contar con un número de habitantes inferior al óptimo en un ambiente dado, que puede ser una iglesia, una escuela o un 
pueblo. Barker utilizó el término "undermanning" para definir esta situación. De la descripción anterior podemos evidenciar que el constructo "falta de personal" aún no era conceptualizado en un contexto exclusivamente laboral. En esa línea, el "understaffing" es una extensión del "undermanning" (Hudson y Shen, 2015). Asimismo, si analizamos la definición brindada, nos percataremos que el criterio de definición es estrictamente cuantitativo ("Manpower Understaffing").

En las décadas siguientes, Perkins (1982), Dietzel y Coursey (1998) delimitan el constructo "falta de personal" o "understaffing" a un escenario laboral, definiéndolo como una situación donde hay muy pocos empleados para completar un trabajo esperado. Srivastava (1974) y Greenberg (1979) la definen como una situación en la que hay muy pocos empleados para cumplir tareas y funciones esenciales de una unidad, una definición más sofisticada. Es en esta perspectiva que Hudson y Shen (2015) también comparten esta última definición.

Como podemos apreciar, a nivel conceptual el constructo "understaffing" o "falta de personal" se concibe cuantitativamente, es decir, en términos del número de trabajadores por debajo del número óptimo para poder realizar las tareas del área a la cual pertenecen. Sin embargo, Hudson y Shen (2015) plantean el carácter multidimensional, por un lado, lo cuantitativo, tal como ha sido concebido en los últimos 60 años con el agregado de que las tareas que se demandan corresponden a tareas claves, es decir, aquellas que son la razón de ser del área. Por ejemplo, una tarea esencial de un área de reclutamiento y selección es poder dotar de nuevo personal que se ajuste a las competencias que ha definido la organización en un tiempo óptimo. A esta dimensión la llamaron "Manpower Understaffing" (Falta de mano de obra).

Por otro lado, la dimensión cualitativa, la cual se refiere a que, si bien un área puede estar numéricamente completa, no es posible asegurar que el personal cuente con las habilidades o experticia requerida para cumplir con las tareas claves del área. En el ejemplo anterior, indistintamente de las causas, la misma área de selección puede contar entre sus integrantes con personal que no cuenta con la experiencia en el sector y no tiene el "know how" de dónde publicar, dónde realizar un "hunting", entre otros. Esto genera, en consecuencia, que la tarea clave no se culmine o demore. A esta dimensión, Hudson y Shen (2015), la llamaron "Expertise Understaffing" (Falta de experticia)

Entonces, podemos convenir que esta situación, sea la dimensión que se manifieste, podría generar situaciones que afectan negativamente el bienestar de las personas del área en la que ocurren. En efecto, Cini, Moreland y Levine (1993), señalaron que la falta de personal está relacionada con resultados negativos a medida que las demandas de un área de trabajo superen los recursos con los que cuentan los integrantes. En esa línea, Dietzel y Coursey (1998) identificaron que la falta de personal se encuentra relacionada negativamente con el "Bienestar Individual" y 
los "Resultados del desempeño del grupo". Aiken, Clarke, Sloane, Sochalski y Silber (2002) identificaron que la "falta de personal" crónica se relaciona con niveles más altos de "Agotamiento" e "Insatisfacción Laboral" en enfermeras.

Asimismo, la presencia de la "falta de personal" también puede influir negativamente en los resultados (productividad) de quienes lo padecen. En esa línea, Clements et al. (2008) y Stapleton et al. (2016) identificaron que la "falta de personal" influye en el "rendimiento" de enfermeras evidenciándose en que sus pacientes recibieron, por parte de enfermeras que percibían "falta de personal", tratamientos ineficientes o erróneos que lo llevaba a mantener cuadros graves de infección o incluso la muerte.

Hudson y Shen (2018) diseñaron la escala "Work Group Understaffing" para medir la "falta de personal", la cual presenta 6 ítems y contiene ambas dimensiones distribuidas con 3 ítems cada una. La construyeron con la finalidad de investigar si los mecanismos mediadores subyacentes a las relaciones entre el "Understaffing", "Rendimiento Individual" y el "Bienestar Individual" eran los mismos para el "Manpower Understaffing” y "Expertise Understaffing”. Para llevar a cabo este estudio, tomaron dos muestras en las que para la dimensión "Manpower Understaffing" la muestra A obtuvo un alfa de .83, mientras que la muestra B obtuvo un alfa de .86. Por el lado de la dimensión "Expertise Understaffing", la muestra A obtuvo un alfa de .80 y la muestra B obtuvo un alfa de .74. La muestra A estuvo conformada por 362 estudiantes universitarios adultos trabajadores, con una edad promedio de 23 años y la muestra B estuvo conformada por 357 trabajadores reclutados de Mechanical Turk de Amazon, con una edad promedio de 32 años, siendo ambas muestras estadounidenses. Cabe precisar que en este estudio no se hace referencia a la validez. También se tiene a Shen, Chang, Cheng y Kim (2019) quienes estudiaron esta escala aplicándolo a una muestra de 382 personas trabajadores de organizaciones tecnológicas de Taiwán como parte de su investigación cuyo objetivo fue identificar cómo los grupos de trabajo enfrentan la falta de personal (en ambas dimensiones), obteniendo en la dimensión "Manpower Understaffing" un alfa de .90 y en la dimensión "Expertise Understaffing" un alfa de .79.

De lo anterior, el presente estudio busca analizar las propiedades psicométricas de la "Escala de Falta de Personal" diseñada por Hudson y Shen (2018) en una muestra de trabajadores de una empresa retail. La importancia del estudio radica en que será la primera herramienta de medición del constructo "falta de personal" en el idioma español y desarrollada en la realidad peruana, permitiendo a futuros investigadores de habla hispana iniciar el estudio de esta variable. Asimismo, a nivel Perú, la presente investigación contribuirá con iniciar el estudio del constructo "falta de personal" más aún para poner sobre la mesa las consecuencias psicológicas sobre los trabajadores de las decisiones que se toman a nivel gerencial basándose en criterios estrictamente financieros en escenarios de incertidumbre económica, recesión, competencia, entre otros. 


\section{MÉTODO}

\section{Participantes}

Colaboraron 201 trabajadores de una empresa del sector retail de electrodomésticos de la ciudad de Lima, Perú. El $22 \%$ son varones y el $78 \%$ son mujeres, con edades entre 18 y 34 años $(M=22.7, D E=3.7)$. Los trabajadores fueron seleccionados a través de un muestro no probabilístico, que en términos de Ñaupas, Mejía, Novoa y Villagomez (2011), la elección de los trabajadores es a criterio del investigador.

\section{Instrumento}

Se empleó la Escala de Falta de Personal de Hudson y Shen (2018). Este instrumento se constituye por dos dimensiones "Manpower Understafing" (Falta de mano de obra) y "Expertise Understafing" (Falta de experticia) y seis ítems, tres ítems por cada dimensión, las cuales son formuladas en positivo (5) y negativo (1). Los ítems 1,2 y 3 constituyen la dimensión "Manpower Understaffing" y los ítems 4, 5 y 6 constituyen la dimensión "Expertise Understaffing". La escala original es de tipo Likert de cinco puntos que va desde "Nunca" (1) hasta "Siempre" (5). De acuerdo con Hudson y Shen (2018) la escala original se aplicó en 2 muestras y solo se reportan valores de alfa de .83 y .86 para la dimensión "Manpower Understaffing" y .80 y .74 en la dimensión "Expertise Understaffing".

Para la traducción del instrumento del inglés al español se utilizó el método "back - translation". Se procedió a realizar la traducción del inglés al español con un traductor certificado. Enseguida, con un segundo traductor certificado, se tradujo del español al inglés replicándose los ítems originales. A continuación, se realizó el análisis de evidencia de validez basado en el contenido mediante la revisión de los ítems por parte de cinco experimentados catedráticos e investigadores en Psicología Organizacional. El resultado de esta validación resultó para los ítems 2 al 6 una $\mathrm{V}$ de Aiken de 1.0 y para el ítem 1 de .80, en los indicadores de "relevancia", "claridad" y "pertinencia". Se determinó que este ítem "Nuestra unidad de trabajo necesita más empleados" era similar a otros, pudiendo implicar redundancia, por esto se decidió retirarlo.

\section{Procedimiento}

Inicialmente se contó con la autorización de la Jefatura de Recursos Humanos de la empresa para la aplicación del instrumento de estudio. Luego se procedió a coordinar con los responsables de sedes y áreas de la organización los horarios en los que los integrantes de sus equipos puedan participar voluntariamente. El ambiente que se utilizó fueron los comedores de las sedes por ser sitios cómodos para el llenado del test, además de estar alejados del ruido exterior. En el lugar, se entregaron las encuestas a los trabajadores en la que se les detalló el objetivo del estudio y el carácter voluntario y se les menciono sobre el anonimato y confidencialidad de la 
información recolectada, logrando el consentimiento informado tal cual lo señala el artículo 24 del código de ética del Colegio de Psicólogos del Perú. Finalmente se procedió a la aplicación de la escala.

\section{Análisis estadístico}

Para el análisis factorial confirmatorio se consideró la naturaleza ordinal de los ítems mediante el cálculo de la matriz de correlaciones policóricas. El estimador usado fue el de mínimos cuadrados ponderados con media y varianza ajustadas (WLSMV), procedimiento recomendado para variables ordinales (Gana y Broc, 2019; Beaducel y Herzberg, 2006). Para la evaluación del ajuste, se consideró los valores $\geq .90$ en CFI y TLI como evidencia a favor del ajuste al modelo (Bentler, 1990; Marsh y Tai Hau, 1996), además de valores $\leq .08$ en RMSEA (MacCallum, Browne y Sugawara, 1996). Por último, para el análisis de confiabilidad, se consideró el método de consistencia interna con el coeficiente omega $(\omega)$, tomándose en cuenta valores mayores a .70. El análisis estadístico se realizó mediante el programa "R" versión 3.1.2 (R Development Core Team, 2007), específicamente con la librería "lavaan" (Rosseel et al., 2018).

\section{RESULTADOS}

Previo al análisis factorial se muestra la matriz de correlaciones policóricas de los ítems de la escala en la Tabla 1. Se observa correlaciones entre .36 y .62.

\section{Tabla 1}

Correlaciones policóricas de los ítems de la escala falta de personal

\begin{tabular}{|c|c|c|c|c|c|}
\hline Ítem & 1 & 2 & 3 & 4 & 5 \\
\hline $\begin{array}{l}\text { 01. No hay suficientes empleados en nuestra unidad de trabajo para completar todas las } \\
\text { tareas de trabajo requeridas. }\end{array}$ & - & & & & \\
\hline $\begin{array}{l}\text { 02. Si en nuestra unidad el trabajo no se termina se debe principalmente a no tener } \\
\text { suficientes empleados para hacerlo. }\end{array}$ & .62 & - & & & \\
\hline 03. En nuestra unidad de trabajo falta personal con conocimientos y habilidades. & .55 & .52 & - & & \\
\hline $\begin{array}{l}\text { 04. Nuestras unidades de trabajo necesitan empleados con diferentes habilidades de las } \\
\text { que actualmente posee el grupo. }\end{array}$ & .36 & .44 & .49 & - & \\
\hline $\begin{array}{l}\text { 05. Si en nuestra unidad el trabajo no se termina, se debe principalmente a que no haya } \\
\text { alguien que sepa cómo hacerlo correctamente. }\end{array}$ & .44 & .51 & .62 & .50 & - \\
\hline$M$ & 3.1 & 2.9 & 2.8 & 3.1 & 2.7 \\
\hline$D E$ & 1.2 & 1.0 & 1.1 & 1.1 & 1.2 \\
\hline
\end{tabular}

Nota. M: media, DE: desviación estándar.

Con respecto a los resultados del análisis factorial confirmatorio, se evaluó la estructura unidimensional del instrumento, encontrándose un ajuste no adecuado, $\chi^{2}(5)=33.46, p<.001, \mathrm{CFI}=.963, \mathrm{TLI}=.925, \mathrm{RMSEA}=.169 . \mathrm{y}$ SRMR $=.046$. Es así como se procede a la evaluación del modelo de dos factores correlacionados 
encontrándose un ajuste adecuado, $\chi^{2}(7)=6.30, p=.177, \mathrm{CFI}=.997$, TLI $=.992$, $\mathrm{RMSEA}=.054$. y SRMR $=.020$. Estos resultados se pueden visualizar en la Tabla 2.

En la Tabla 2 se presenta los resultados de las cargas factoriales para el modelo de dos factores correlacionados, los cuales se encuentran entre .62 y .81. Asimismo, las correlaciones entre dimensiones presentan valores entre .76 y .82., indicando una relación significativa.

\section{Tabla 2}

Cargas factoriales de la solución estandarizada del análisis factorial confirmatorio para el modelo final

\begin{tabular}{|c|c|c|}
\hline Ítem & F1 & F2 \\
\hline $\begin{array}{l}\text { 01. No hay suficientes empleados en nuestra unidad de trabajo para completar todas las tareas de } \\
\text { trabajo requeridas. }\end{array}$ & .77 & \\
\hline $\begin{array}{l}\text { 02. Si en nuestra unidad el trabajo no se termina se debe principalmente a no tener suficientes em- } \\
\text { pleados para hacerlo. }\end{array}$ & .81 & \\
\hline 03. En nuestra unidad de trabajo falta personal con conocimientos y habilidades. & & .81 \\
\hline $\begin{array}{l}\text { 04. Nuestras unidades de trabajo necesitan empleados con diferentes habilidades de las que actual- } \\
\text { mente posee el grupo. }\end{array}$ & & .62 \\
\hline $\begin{array}{l}\text { 05. Si en nuestra unidad el trabajo no se termina, se debe principalmente a que no haya alguien que } \\
\text { sepa cómo hacerlo correctamente. }\end{array}$ & & .76 \\
\hline \multicolumn{3}{|l|}{ Correlaciones entre factores } \\
\hline F1. Manpower Understaffing & - & \\
\hline F2. Expertise Understaffing & .82 & - \\
\hline
\end{tabular}

Finalmente, se realizó el análisis de la confiabilidad del modelo de dos factores correlacionados de la falta de personal, a través del análisis de la consistencia interna utilizando el coeficiente omega $(\omega)$. El resultado obtenido luego del análisis fue de .77 en la dimensión "falta de mano de obra" y de .78 para la dimensión "falta de experticia".

\section{DISCUSIÓN}

El objetivo de la investigación ha sido analizar las propiedades psicométricas de la escala "Falta de personal" de Hudson y Shen (2018), relacionadas a las evidencias de validez basado en el contenido y en la estructura interna, además del análisis de confiabilidad.

En lo que se refiere a la evidencia de validez basado en la estructura interna, se realizó el análisis factorial confirmatorio de dos modelos: el modelo unidimensional y el modelo de dos factores correlacionados, siendo este último el que presenta un ajuste adecuado. Este resultado se encuentra en sintonía con el marco teórico definido por Hudson y Shen (2015). Dentro de este procedimiento se retiró el ítem 1, "Nuestra unidad de trabajo necesita más empleados", a partir de la evaluación de 
jueces y de la similitud con el ítem 2, "No hay suficientes empleados en nuestra unidad de trabajo para completar todas las tareas de trabajo requeridas.". Esto se realizó a fin de evitar la redundancia y las distorsiones que esta implica.

Respecto a la confiabilidad, determinado por el método de consistencia interna se utilizó el coeficiente omega, en la que el modelo de dos factores correlacionados de la falta de personal presenta un valor mayor a .70, el cual se considera aceptable (Campo-Arias y Oviedo, 2008). De acuerdo con Anderson y Gerbing (1988), la ventaja del coeficiente omega es ser menos restrictivo que el coeficiente alfa. El presente trabajo es el primero en reportar este coeficiente a diferencia de los de Hudson y Shen (2018) y Shen, Chang, Cheng y Kim (2019) en los que utilizaron el coeficiente alfa.

Una fortaleza del estudio corresponde al análisis estructural que se realizó haciendo consideración de la naturaleza ordinal de los ítems. Esto se realizó utilizando la matriz es de correlaciones políticas, además del estimador WLSMV. Además, estos resultados se usaron para el cálculo del coeficiente de consistencia interna omega. Asimismo, consideramos que un importante aporte del estudio es analizar la multidimensionalidad del constructo "falta de personal", es decir, no solo enfocarlo desde un punto de vista exclusivamente cuantitativo, diferenciándolo de otros estudios como los de Treanor y Cotch (1990), Pavinne, Van-Nam y Thepchai (2020).

Es preciso mencionar que, dadas las características descritas de los participantes, la generalización o extensión de estos resultados se circunscriben a un sector de retail. Además, como limitación en cuanto el número de participantes, este no permite hacer el estudio del funcionamiento del instrumento en distintos grupos, es decir un análisis de invarianza factorial. También, se debe agregar que la recolección de datos se dio mediante escalas de autoinforme, por lo que las respuestas podrían contener distorsiones por deseabilidad social, entre otros.

Finalmente, en esta investigación, y en razón a los hallazgos mostrados, se ofrece una primera aproximación a la operacionalización del constructo "Falta de personal" en el Perú. Se recomienda realizar replicaciones en las que se continúe revisando el análisis de las propiedades psicométricas en otros sectores laborales.

\section{Conflictos de interés}

Los autores declaran que no existen conflictos de interés en la elaboración del manuscrito.

\section{Financiamiento}

Autofinanciado. 


\section{REFERENCIAS}

Anderson, J. C. y Gerbing, D. W. (1988). Structural Equation Modeling in Practice: A Review and Recommended Two-Step Approach. Psychological Bulletin, 103(3), 411-423. https://doi.org/10.1037/0033-2909.103.3.411

Aiken, L. H., Clarke, S. P., Sloane, D. M., Sochalski, J. y Silber, J. H. (2002). Hospital nurse staffing and patient mortality, nurse burnout, and job dissatisfaction. Journal of the American Medical Association, 288(16), 1987-1993. https://doi.org/10.1001/ jama.288.16.1987

Barker, R. (1968). Ecological psychology: Concepts and methods for studying the environment of human behavior. Stanford, CA: Standford University Press, 1968. 242 p.

Beaducel, A. y Herberg, P. Y. (2006). On the performance of maximum likelihood versus means and variance adjusted weighted least squares estimation in CFA. Structural Equation Modeling: A Multidisciplinary Journal, 13 (29), 186-203. https://doi. org/10.1207/s15328007sem1302_2

Bentler, P. (1990). Comparative Fit Indices in Structural Models. UCLA: Department of Statistics, UCLA. Retrieved from https://escholarship.org/uc/item/2mk8r49v

Campo-arias, A. y Oviedo, H. (2008). Disponible en: http://www.redalyc.org/articulo. oa?id=42210515. Revista de Salud Pública, 10, 831-839.

Cini, M.A., Moreland, R.L. y Levine, J.M. (1993). Group staffing levels and responses to prospective and new group members. Journal of Personality and Social Psychology, 65(4), 723-734. doi:10.1037/0022-3514.65.4.723

Clements, A., Halton, K., Graves, N., Pettitt, A., Morton, A., Looke, D. y Whitby, M. (2008). Overcrowding and understaffing in modern health-care systems: key determinants in meticillin-resistant Staphylococcus aureus transmission. The Lancet Infectious Diseases, 8(7), 427-434. https://doi.org/10.1016/S1473-3099(08)70151-8

Dietzel, L. C. y Coursey, R. D. (1998). Predictors of emotional exhaustion among nonresidential staff persons. Psychiatric Rehabilitation Journal, 21(4), 340-348. https://doi. $\operatorname{org} / 10.1037 / \mathrm{h} 0095290$

Gana, K \& Broc, G. (2019). Structural Equation Modeling with lavaan. Wiley, 304 p.

Greenberg, C. I. (1979). Toward an integration of ecological psychology and industrial psychology: Undermanning theory, organization size, and job enrichment. Environmental Psychology and Nonverbal Behavior, 3(4), 228-242. https://doi.org/10.1007/BF01127366

Herrera, Y. C., Betancur, J., Jiménez, N. L. S. y Martínez, A. M. (2017). Revista Electrónica Psyconex. Revista Electrónica Psyconex, 9(14), 1-13. https://aprendeenlinea.udea. edu.co/revistas/index.php/Psyconex/article/view/328547/20785360

Hudson, C. K. y Shen, W. (2015). Understaffing: An under-researched phenomenon. Organizational Psychology Review, 5(3), 244-263. https://doi.org/10.1177/2041386615576016

Hudson, C. K. y Shen, W. (2018). Consequences of work group manpower and expertise understaffing: A multilevel approach. Journal of Occupational Health Psychology, 23(1), 85-98. https://doi.org/10.1037/ocp0000052 
MacCallum, R. C., Browne, M. W. y Sugawara, H. M. (1996). Power analysis and determination of sample size for covariance structure modeling. Psychological Methods, 1(2), 130-149. https://doi.org/10.1037/1082-989X.1.2.130

Marsh, H. W. y Hau, K. T. (1996). Assessing goodness of fit: Is parsimony always desirable? Journal of Experimental Education, 64(4), 364-390. https://doi.org/10.1080/002 20973.1996.10806604

Ñaupas, H., Mejía, E., Novoa, E. y Villagomez, A. (2011). Metodología de la investigación científica y asesoramiento de tesis. Lima: Editorial Humberto Naupas Paitan.

PerkinS, D. V. (1982). Individual differences and task structure in the performance of a behavior setting: An experimental evaluation of Barker's manning theory. American Journal of Community Psychology, 10(6), 617-634. https://doi.org/10.1007/ BF01312595

R Development Core Team, 2007. R: a language and environment for statistical computing. R Foundation for Statistical Computing, Vienna, Austria, ISBN 3-900051-07-0, URL http://www.R-project.org

Rerkjirattikal, P., Huynh, V.-N., Olapiriyakul, S., \& Supnithi, T. (2020). A Goal Programming Approach to Nurse Scheduling with Individual Preference Satisfaction. Mathematical Problems in Engineering, 2020, 1-11. https:// doi:10.1155/2020/2379091

Rivera, A. (2017). Efecto de la carga de trabajo en el desempeño de los trabajadores. Universidad Militar Nueva Granada, 18. http://repository.unimilitar.edu.co/bitstream/10654/16216/1/RiveraSanchezAngelaBibiana2017.pdf

Rodríguez, A. (2010). Estructuras flexibles de costos para enfrentar la globalización y la competitividad en las empresas. Revista de la Facultad de Ciencias Contables UNMSM, 17(33), 19-26. https://doi.org/10.15381/quipu.v17i33.4546

Rosseel, Y., Oberski, D., Byrnes, J., Vanbrabant, L., Savalei, V., Merkle, E. y Chow, M. (2018). Package 'lavaan’ 0.6-2. Recuperado de https:// cran.r-project.org/web/packages/lavaan/lavaan.pdf

Shen, W., Chang, K., Cheng, K. T. y Kim, K. Y. (2019). What to do and what works? Exploring how work groups cope with understaffing. Journal of Occupational Health Psychology, 24(3), 346-358. https://doi.org/10.1037/ocp0000129

Srivastava, R. K. (1974). Undermanning theory in the context of mental health care environments. In D. H. Carson (Ed.), Man environment interactions, Part II (pp. 245 258). Stroudsburg, PA: Dowden, Hutchinson y Ross.

Stapleton, P. J. M., Murphy, M., McCallion, N., Brennan, M., Cunney, R. y Drew, R. J. (2016). Outbreaks of extended spectrum beta-lactamaseproducing Enterobacteriaceae in neonatal intensive care units: A systematic review. Archives of Disease in Childhood: Fetal and Neonatal Edition, 101(1), F72-F78. https://doi.org/10.1136/archdischild-2015-308707

Treanor, J., \& Cotch, K. (1990). Staffing of adult psychiatric inpatient facilities. Hospital and Community Psychiatry, 41, 545-549. https:// doi:10.1176/ps.41.5.545

Varian, H. (1998). Análisis microeconómico. Antoni Bosch. 